\title{
Transfer of global wellposedness \\ from nonlinear Klein-Gordon equation \\ to nonlinear Schrödinger equation
}

\author{
Kenji NAKANISHI
}

(Received November 17, 2007)

\begin{abstract}
We discuss relations between the nonlinear Klein-Gordon equation and the nonlinear Schrödinger equation in view of the global wellposedness in the energy space and $L^{2}$. In some critical cases, we show that the global wellposedness for the former equation with some uniform bounds implies that for the latter.

Key words: nonlinear Klein-Gordon equation, nonlinear Schrödinger equation, wellposedness, nonrelativistic limit.
\end{abstract}

\section{Introduction}

This article is an extension of the comment the author gave at Jun Kato's talk in the conference. It was in short that Strichartz-type spacetime estimates for the Schrödinger equation with the $L^{2}$ scaling follow from the same estimates for the Klein-Gordon equation with $H^{1}$ initial data.

Kato's talk was about his recent work with Tohru Ozawa on the endpoint Strichartz estimate in $L_{t}^{2} L_{r}^{\infty} L_{\theta}^{q}\left(\mathbb{R}^{1+2}\right)$ for the Klein-Gordon equation, and the above comment was to point out that their result immediately implies the same bound for the Schrödinger equation with $L^{2}$ initial data.

Although that observation seems almost trivial, the author decided to present it here, with some nonlinear contexts, at least for the following three reasons.

(1) It did not seem to be recognized very well, even among those experts who attended the conference, despite the popularity of the Strichartz estimate for both equations.

(2) Some nonlinear versions have very interesting implications for much deeper questions, although they need some assumptions to be proved.

(3) Such an article seems to be most suited for this sort of proceedings, where the contributions should be original and related to the con-

2000 Mathematics Subject Classification : 35L70, 35Q55, 35B40, 35B25. 
ference, whereas it is not customary in mathematics to publish final results in proceedings.

We will discuss the relation between the nonlinear Klein-Gordon equation (NLKG) and the nonlinear Schrödinger equation (NLS), given in the following form:

$$
\begin{aligned}
& u_{t t}-\Delta u+u=f(u):=-\mu|u|^{\alpha} u, \\
& 2 i u_{t}-\Delta u=f(u),
\end{aligned}
$$

where $u(t, x): \mathbb{R}^{1+d} \rightarrow \mathbb{C}$ is the unknown function, $\alpha>0$ and $\mu \geq 0$ are constants. The scaling invariance of $f(u)$ is essential throughout the paper. We will consider mainly the two critical cases:

$$
\alpha=\frac{4}{d}, \frac{4}{d-2} \text {. }
$$

The invariant scaling for NLS in each case leaves invariant the $L_{x}^{2}$ norm and the $\dot{H}_{x}^{1}$ norm respectively of the solutions.

Our main claim is that we can transfer space-time estimates from NLKG to NLS, and for some appropriate norms, it also transfers global wellposedness together with scattering. To state our results precisely, we need a few basic concepts.

First, the NLKG has the following energy conserved

$$
E_{K}(u ; t):=\frac{1}{2} \int_{\mathbb{R}^{d}}|\dot{u}|^{2}+|\nabla u|^{2}+|u|^{2}+F(u) d x,
$$

where the function $F$ is defined by

$$
F(\varphi):=\frac{2 \mu}{\alpha+2}|\varphi|^{\alpha+2} .
$$

Similarly, the NLS conserves the energy

$$
E_{S}(u ; t):=\frac{1}{2} \int_{\mathbb{R}^{d}}|\nabla u|^{2}+F(u) d x,
$$

and also the $L^{2}$ norm

$$
M_{S}(u ; t):=\int_{\mathbb{R}^{d}}|u|^{2} d x .
$$

The corresponding quantity for the NLKG is defined by

$$
M_{K}(u ; t):=\int_{\mathbb{R}^{d}} \operatorname{Im}(\dot{u} \bar{u}) d x,
$$


which is not sign definite. Linear combination of $E$ and $M$ makes a family of conserved quantities. We denote in particular

$$
\begin{aligned}
E_{K}^{\prime}(u ; t) & :=E_{K}(u ; t)-M_{K}(u ; t) \\
& =\frac{1}{2} \int_{\mathbb{R}^{d}}|\dot{u}-i u|^{2}+|\nabla u|^{2}+F(u) d x,
\end{aligned}
$$

which is still positive definite and more related to the Schrödinger energy. We will often drop the time $t$ from the above notation, provided that the quantity is conserved.

Next we introduce some invariance properties of function spaces adapted for NLS.

Definition 1.1 For any $u \in \mathcal{S}^{\prime}\left(\mathbb{R}^{1+d}\right), \beta \in \mathbb{R}$ and $\lambda>0$, we define the space-time function $P_{\lambda}^{\beta} u$ by

$$
\left(P_{\lambda}^{\beta} u\right)(t, x)=\lambda^{\beta} u\left(\lambda^{2} t, \lambda x\right) .
$$

Let $X \subset \mathcal{S}^{\prime}\left(\mathbb{R}^{1+d}\right)$ be a semi-normed space. We say $X$ is parabolically homogeneous of degree $\beta \in \mathbb{R}$ if

$$
\left\|P_{2}^{\beta} u\right\|_{X}=\|u\|_{X},
$$

for all $u \in X$. We say $X$ is gauge invariant if

$$
\left\|e^{i t \lambda} u\right\|_{X}=\|u\|_{X}
$$

for all $u \in X$ and $\lambda>0$. We say $X$ has the Fatou property if for any bounded sequence $u_{n} \in X$ convergent in $\mathcal{S}^{\prime}\left(\mathbb{R}^{1+d}\right)$, the limit $u_{\infty}$ is in $X$ and

$$
\left\|u_{\infty}\right\|_{X} \leq \liminf _{n \rightarrow \infty}\left\|u_{n}\right\|_{X}
$$

The last condition is required for the transfer argument, and satisfied if $X$ is a dual of some normed space $Y$ in which $\mathcal{S}\left(\mathbb{R}^{1+d}\right)$ is dense. For example, $L_{t}^{p} L_{x}^{q}$ and $L_{t}^{p} \dot{B}_{q, r}^{\sigma}$, where $\dot{B}_{q, r}^{\sigma}$ denotes the homogeneous Besov space, are parabolically homogeneous of degree $2 / p+d / q$ and $2 / p+d / q-\sigma$ respectively ${ }^{1}$. Moreover, they are gauge invariant, and for $p, q, r>1$, they have the Fatou property.

\footnotetext{
${ }^{1}$ We need to choose appropriate norms for the Besov spaces to have the equality in the definition of homogeneity, for example using the dyadic decomposition constructed by binary dilation, as usual [2].
} 
Finally we denote the frequency localization for $\varphi \in \mathcal{S}^{\prime}\left(\mathbb{R}^{d}\right)$ at $R>0$ by

$$
\varphi_{<R}=\mathcal{F}^{-1}\left[\chi\left(\frac{\xi}{R}\right)(\mathcal{F} \varphi)(\xi)\right]
$$

where $\mathcal{F}$ denotes the Fourier transform on $\mathcal{S}^{\prime}\left(\mathbb{R}^{d}\right)$, and $\chi \in C_{0}^{\infty}\left(\mathbb{R}^{d}\right)$ is arbitrarily chosen and fixed such that $\chi(\xi)=1$ for $|\xi|<1 / 2$ and $\chi(\xi)=0$ for $|\xi|>2$. The above notation extends to the space-time functions in the obvious way.

Now we can state our main results.

\section{1. $\quad L^{2}$ critical case}

First for the $L^{2}$ critical case $\alpha=4 / d$, we have

Theorem 1.2 Let $d \in \mathbb{N}, \alpha=4 / d$ and $\mu \geq 0$. Let $X \subset \mathcal{S}^{\prime}\left(\mathbb{R}^{1+d}\right)$ be a semi-normed space parabolically homogeneous of degree $d / 2$ and gauge invariant with the Fatou property. Suppose that for any finite energy solution u of $N L K G$ (1.1) we have

$$
\left\|u_{<\delta}\right\|_{X} \leq C_{X}\left(E_{K}(u)\right),
$$

for some fixed $\delta>0$ and $C_{X}:[0, \infty) \rightarrow[0, \infty)$ continuous. In the case $\mu>$ 0 , suppose that the above holds also with $X=L_{t}^{p} L_{x}^{q}$ for some $p \in[2, \infty)$.

Then for any $L^{2}\left(\mathbb{R}^{d}\right)$ initial data, we have a unique global solution $w \in$ $C\left(\mathbb{R} ; L^{2}\right) \cap L^{2+4 / d}\left(\mathbb{R}^{1+d}\right)$ for $N L S(1.2)$, together with the scattering

$$
\lim _{t \rightarrow \pm \infty}\left\|w(t)-w_{ \pm}(t)\right\|_{L^{2}\left(\mathbb{R}^{d}\right)}=0
$$

for some free solutions $w_{ \pm}$, and the transferred bound

$$
\|w\|_{X} \leq C_{X}\left(M_{S}(w)\right),
$$

for the same function $C_{X}$ as above.

It is well known that every finite energy initial data leads to a unique global solution for NLKG in the above case.

In the linear case $\mu=0$, the Strichartz estimate gives

$$
\|u\|_{L_{t}^{p} B_{q, 2}^{\sigma}} \lesssim E_{K}(u)^{1 / 2}, \quad \sigma=1-\frac{1+4 / d}{p}>0,
$$

when $L_{t}^{p} L_{x}^{q}$ is parabolically homogeneous of degree $d / 2$ and $2 \leq p \leq \infty$, except for the endpoint $(p, q, d)=(2, \infty, 2)$. In this case, the conclu- 
sion of the theorem is just the standard Strichartz estimate for the linear Schrödinger.

However the above theorem itself is valid at the endpoint too. In particular, the Kato-Ozawa endpoint Strichartz estimate for the free KleinGordon

$$
\|u\|_{L_{t}^{2} L_{r}^{\infty} L_{\theta}^{q}\left(\mathbb{R}^{1+2}\right)} \leq C q^{1 / 2} E_{K}(u)^{1 / 2} \quad(q<\infty),
$$

implies the same bound for the free Schrödinger

$$
\|w\|_{L_{t}^{2} L_{r}^{\infty} L_{\theta}^{q}\left(\mathbb{R}^{1+2}\right)} \leq C q^{1 / 2} M_{S}(w)^{1 / 2} \quad(q<\infty) .
$$

The latter estimate was previously known only for $q$ close to 2 , by the result of Tao [27]. Indeed it was shown in [17] that one cannot achieve the above estimate for $q>6$ by using Tao's approach. Moreover Kato-Ozawa's proof, following the argument in [17] for the three dimensional wave equation, seems simpler than Tao's.

Concerning the nonlinear case $\mu>0$, the uniform bound (1.15) is expected to be true for the Strichartz norm $L_{t}^{p} L_{x}^{q}$, but unfortunately not yet proven. Moreover, the global wellposedness of NLS in $L^{2}$ still remains to be an open problem. The uniform global bound on the Strichartz norms has been proven only for the higher power $4 / d<\alpha \leq 4 /(d-2)$, which holds for the whole frequency as in (1.18). See [20, 21, 22] for $\alpha=4 /(d-2), d \leq 2$, and the general case, respectively. The finiteness had been known before for $d \geq 3$ with $\alpha<4 /(d-2)$, see e.g. [12], which is sufficient for the scattering of NLKG, but not transferable to NLS.

In the critical case $\alpha=4 / d$ for the NLKG, it is known that for any free solution with finite energy, there exists a unique solution of NLKG which has globally finite $L_{t, x}^{2+4 / d}$ norm and approaches the free one as $t \rightarrow \infty$. For small energy data, (1.18) (and so including all the other conclusions) is an immediate consequence of the standard iteration by the Strichartz estimate. However the asymptotic completeness, which is equivalent to finiteness of the $L_{t, x}^{2+4 / d}$ norm of all solutions, remains an open question.

As for NLS, there has been recently intensive study on the global wellposedness for $\alpha=4 / d$, but it is so far achieved only for small data [29], or in more regular space $[5,7,8,11,30,9,10]$, or under the radial symmetry $[15,16]$. The above theorem suggests a totally different approach, but also implying that global estimates for the NLKG with $\alpha=4 / d$ in $H^{1}$ can be just as difficult as the same problem for NLS in $L^{2}$. 


\section{2. $H^{\mathbf{1}}$ critical case}

Next we consider the $H^{1}$ critical case $\alpha=4 /(d-2), d>2$. We recall that the modified energy $E_{K}^{\prime}=E_{K}-M_{K}$ was defined in (1.9). For any Banach space $X$, we denote by $w$-X the same space endowed with the weak topology.

Theorem 1.3 Let $d \geq 3$ and $\alpha=4 /(d-2)$ with $\mu \geq 0$. Let $X \subset \mathcal{S}^{\prime}\left(\mathbb{R}^{1+d}\right)$ be a semi-normed space parabolically homogeneous of degree $d / 2-1$ and gauge invariant with the Fatou property. Suppose that for any finite energy solution $u$ of $N L K G(1.1)$ we have

$$
\left\|u_{<\delta}\right\|_{X} \leq C_{X}\left(E_{K}^{\prime}(u)\right),
$$

for some fixed $\delta>0$ and $C_{X}:[0, \infty) \rightarrow[0, \infty)$ continuous. Then for any $\dot{H}^{1}\left(\mathbb{R}^{d}\right)$ initial data, there exists a weak global solution $w$ of NLS (1.2) satisfying $w \in C\left(\mathbb{R} ; w-\dot{H}^{1}\left(\mathbb{R}^{d}\right)\right)$ and

$$
\|w\|_{X} \leq C_{X}\left(E_{S}(w ; 0)\right),
$$

with the same $C_{X}$ as above.

Moreover, if the above assumption holds with $X=L_{t}^{p} \dot{H}_{q}^{1}$ for some $p \in$ $[2, \infty)$, then we have the global wellposedness for the NLS, together with the scattering

$$
\lim _{t \rightarrow \pm \infty}\left\|w(t)-w_{ \pm}(t)\right\|_{\dot{H}^{1}\left(\mathbb{R}^{d}\right)}=0,
$$

for some free solutions $w_{ \pm}$

In the linear case $\mu=0$, the Strichartz estimate gives

$$
\left\|u_{<1}\right\|_{L_{t}^{p} \dot{H}_{q}^{1}} \lesssim E_{K}^{\prime}(u)^{1 / 2}
$$

when $L^{p} \dot{H}_{q}^{1}$ is parabolically homogeneous of degree $d / 2-1$ and $2 \leq p \leq \infty$ [18]. The last assertion in the theorem, namely the global wellposedness with scattering for the $\dot{H}^{1}$ critical NLS, was proved for the radial data in $[4,28,14]$, and for general data in $[6,24,31]$, but the proofs have been much more complicated than those for the critical wave and NLKG equations $[13,25,1,20]$. The above theorem suggests a possibility of completely different proof.

For the critical NLKG, the following uniform bound was proven in [20]:

$$
\|u\|_{L_{t}^{p} B_{q, 2}^{\sigma}} \leq C\left(E_{K}(u)\right),
$$


for the same left hand side as in (1.18), which is stronger than that in (1.24), but the bound depends on the full energy $E_{K}(u)$. It is unfortunately not known if the estimate of the type (1.24) holds or not.

\subsection{Dyadic transition estimate}

Next we consider $L_{t}^{\infty}$-type bounds which leads to the global wellposedness in the critical case. Trivially, $X=L_{t}^{\infty} \dot{H}_{x}^{1}$ satisfies the assumption in the above theorem, but it is not sufficient even for the local unique existence. The reason is roughly speaking that the $L_{t}^{\infty}$ bound does not contain any finiteness information in the time direction, which is crucial for the wellposedness in the critical case. Hence we have to blend some finiteness information in time. Here is a candidate, which seems interesting by itself. Heuristically, it is designed to measure the duration in logarithmic scale for the transition of the nonlinear solution from the initial state to the scattering states at $t= \pm \infty$.

Definition 1.4 For any Banach space $B \subset \mathcal{S}^{\prime}\left(\mathbb{R}^{d}\right)$ and $p \in(1, \infty)$, we define the semi-norms $S_{1}^{p} B$ and $S_{\infty}^{p} B$ as follows. For any $u \in C^{1}\left(\mathbb{R} ; \mathcal{S}^{\prime}\left(\mathbb{R}^{d}\right)\right)$ and $T \in \mathbb{R}$, let $u^{T}$ be the solution to the free Klein-Gordon

$$
\left[\partial_{t}^{2}-\Delta+1\right] u^{T}=0, \quad u^{T}(T)=u(T), \dot{u}^{T}(T)=\dot{u}(T) .
$$

We define the $S_{1}^{p} B$ semi-norm of $u$ by

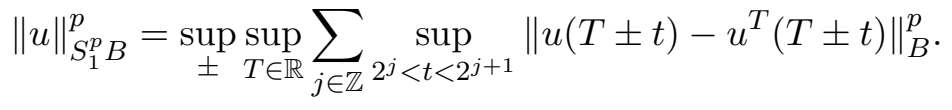

Similarly for any $w \in C^{0}\left(\mathbb{R} ; \mathcal{S}^{\prime}\left(\mathbb{R}^{d}\right)\right)$ and $T \in \mathbb{R}$, let $w^{T}$ be the solution to the free Schrödinger

$$
\left[2 i \partial_{t}-\Delta\right] w^{T}=0, \quad w^{T}(T)=w(T) .
$$

We define the $S_{\infty}^{p} B$ semi-norm of $w$ by

$$
\|w\|_{S_{\infty}^{p} B}^{p}=\sup _{ \pm} \sup _{T \in \mathbb{R}} \sum_{j \in \mathbb{Z}^{j}<t<2^{j+1}} \sup _{j}\left\|w(T \pm t)-w^{T}(T \pm t)\right\|_{B}^{p} .
$$

The subscripts 1 and $\infty$ stand for the propagation speeds of the referred equations. By definition, those semi-norms vanish exactly on the set of free solutions. Our next theorem deals with transfer of the above semi-norms from NLKG to NLS in the $H^{1}$ critical case. 
Theorem 1.5 Let $d \geq 3$ and $\alpha=4 /(d-2)$ with $\mu \geq 0$. Assume that every solution $(u, \dot{u}) \in C\left(\mathbb{R} ; H^{1} \times L^{2}\right)$ of $N L K G$ (1.1) satisfies

$$
\left\|u_{<\delta}\right\|_{S_{1}^{p} L^{2^{*}}} \leq C\left(E_{K}^{\prime}(u)\right)
$$

for some fixed $p \in(1, \infty), \delta>0$ and $C:[0, \infty) \rightarrow[0, \infty)$ continuous, where $2^{*}=2 d /(d-2)=p+2$ is the Sobolev critical exponent.

Then for any $\varphi \in \dot{H}^{1}\left(\mathbb{R}^{d}\right)$, there exists a unique global solution $w$ of $N L S(1.2)$ satisfying $w \in C\left(\mathbb{R} ; \dot{H}^{1}\left(\mathbb{R}^{d}\right)\right)$ and

$$
\|w\|_{S_{\infty}^{p} L_{x}^{2 *}} \leq C\left(E_{S}(w)\right)
$$

where $C$ and $p$ are the same as above. Moreover we have the scattering

$$
\lim _{t \rightarrow \pm \infty}\left\|w(t)-w_{ \pm}(t)\right\|_{\dot{H}^{1}\left(\mathbb{R}^{d}\right)}=0
$$

for some free Schrödinger solutions $w_{ \pm}$.

As mentioned in the previous theorem, the global wellposedness with scattering for the NLS has been already proved, but in very complicated ways. The above theorem suggests a possibility of another simpler proof.

The uniform bound on the Strichartz norms proved in [20] implies a uniform bound on the $S_{1}^{2}$ norm in terms of $E_{K}(u)$, which is true in the other case of power. As in the case of Strichartz norms, the difference from the assumption (1.30) is whether the bound is given in terms of $E_{K}$ or $E_{K}^{\prime}$.

Theorem 1.6 Let $d \geq 3$ and $4 / d<\alpha \leq 4 /(d-2)$ with $\mu \geq 0$. Then for any finite energy solution $u$ of $N L K G(1.1)$, we have

$$
\left\|u_{<1}\right\|_{S_{1}^{2} L^{2^{*}}} \leq C\left(E_{K}(u)\right)
$$

The same estimate for NLS follows from the uniform bounds given in $[3,6]$.

Theorem 1.7 Let $d \geq 3$ and $4 / d<\alpha \leq 4 /(d-2)$ with $\mu \geq 0$. Then for any finite energy solution $w$ of NLS (1.2), we have

$$
\|w\|_{S_{\infty}^{2} L^{2^{*}}} \leq C\left(E_{S}(w)+M_{S}(w)\right)
$$

where $M_{S}(w)$ is not necessary when $\alpha=4 /(d-2)$.

We will prove linear estimates which connects the Strichartz bounds and the dyadic transition norms. Strictly speaking in the above theorems, 
we should restrict the solution class to ensure the uniqueness in the critical case $\alpha=4 /(d-2)$, where the unconditional uniqueness of finite energy solution is not known (see $[26,23,19]$ for partial results).

The estimates (1.33) and (1.34) look interesting by themselves. The summability for $j \rightarrow-\infty$ and $j \rightarrow \infty$ comes from different reasons. The former is due to the convergence at $t=0$, but in general the nonlinear solution approaches another free solution as $t \rightarrow \infty$, so the subtraction of the initial free solution does not help decay for $t \rightarrow \infty$. The summability for $j \rightarrow \infty$ comes purely from the dispersion in $L_{x}^{2^{*}}$ at $t \rightarrow \infty$, both for the initial free solution and the asymptotic free solution. Moreover, by time translation invariance, the transition from the initial approximation regime to the dispersion regime can take place at any time. The estimates imply that the duration of the transition (or "scattering") is uniformly bounded in the logarithmic time scale. In other words, after the nonlinear solution $u$ leaves from the initial free solution $u^{0}$, both $u$ AND $u^{0}$ have to start dispersing rather soon. Notice that the logarithmic scale is necessary at least for the limit because of the scaling invariance.

The semi-norm $S_{1}^{p} L^{2^{*}}$ was inspired by the a priori bound for the $H^{1}$ critical NLKG:

$$
\sum_{j \in \mathbb{Z}^{2}} \sup _{2^{j}<t<2^{j+1}} \int_{|x|<R|t|}|u(t, x)|^{2^{*}} d x \leq C\left(E_{K}(u), R\right) .
$$

More precisely, the above estimate was derived in [20] globally for the (massless) wave equation, and locally on $t \in[0,1]$ for NLKG, as a quantitative version of the $L^{2^{*}}$ non-concentration argument in [25]. The global version for NLKG follows from the uniform bound on the Strichartz norms given in [20].

However the above estimate cannot hold if one replaces $E_{K}$ on the right with $E_{K}^{\prime}$, even in the linear case $\mu=0$. That is because if the estimate remained true, then it would imply the same bound for NLS without the restriction $|x|<R|t|$, which is clearly false for $t \rightarrow 0$, or $j \rightarrow-\infty$.

\section{Formal limit from NLKG to NLS}

In this section we recall how NLS can be derived as a singular limit from NLKG, using a combination of rescaling and phase change.

Let $u$ be a solution to NLKG (1.1), and $v:=e^{-i t} u$. Then the mass 
term is removed and we get the equation for $v$

$$
v_{t t}+2 i v_{t}-\Delta v=f(v),
$$

where we used the gauge invariance of $f$. Next we perform the parabolic rescaling $w:=P_{c}^{2 / \alpha} v$. Then we have $u=e^{i t} c^{-2 / \alpha} w\left(t / c^{2}, x / c\right)$ and the equation is transformed into

$$
c^{-2} w_{t t}+2 i w_{t}-\Delta w=f(w) .
$$

In the limit $c \rightarrow \infty$, formally the first term disappears, and we get the NLS (1.2) for the limit of $w$.

\section{Limit of the energy}

Next we investigate how the energy behaves in the above limit. Let $v=e^{-i t} u$ and $w=P_{c}^{2 / \alpha} v$ as above. Then we have

$$
\begin{aligned}
E_{K}(u) & =\frac{1}{2} \int_{\mathbb{R}^{d}}|\dot{v}+i v|^{2}+|\nabla v|^{2}+|v|^{2}+F(v) d x \\
& =c^{d-4 / \alpha}\left[\frac{1}{2} \int_{\mathbb{R}^{d}}\left|\frac{\dot{w}}{c}+i w\right|^{2}+\left|\frac{\nabla w}{c}\right|^{2}+|w|^{2}+c^{-2} F(w) d x\right] .
\end{aligned}
$$

We observe that in the special case $\alpha=4 / d$, the right hand side formally converges to $M_{S}(w)$ as $c \rightarrow \infty$.

For the mass we have

$$
\begin{aligned}
M_{K}(u) & =\int_{\mathbb{R}^{d}} \operatorname{Im}(\dot{v} \bar{v})+|v|^{2} d x \\
& =c^{d-4 / \alpha} \int_{\mathbb{R}^{d}} c^{-2} \operatorname{Im}(\dot{w} \bar{w})+|w|^{2} d x,
\end{aligned}
$$

which formally converges to $M_{S}(w)$ as $c \rightarrow \infty$ if $\alpha=4 / d$. For the reduced energy we have

$$
\begin{aligned}
E_{K}^{\prime}(u) & =\frac{1}{2} \int_{\mathbb{R}^{d}}|\dot{v}|^{2}+|\nabla v|^{2}+F(v) d x \\
& =c^{d-2-4 / \alpha}\left[\frac{1}{2} \int_{\mathbb{R}^{d}}|\dot{w} / c|^{2}+|\nabla w|^{2}+F(w) d x\right] .
\end{aligned}
$$

In the special case $\alpha=4 /(d-2)$, the above converges formally to the non-relativistic energy $E_{S}(w)$ as $c \rightarrow \infty$. 
In the later arguments, we will choose a sequence of initial data depending on the parameter $c \rightarrow \infty$, such that the above formal limits can be justified together with convergence of the solutions.

\subsection{Proof for $L^{2}$ critical case}

In this section we prove the transfer Theorem 1.2 in the $L^{2}$ critical case $\alpha=4 / d$. First we consider initial data $\varphi \in H^{1}\left(\mathbb{R}^{d}\right)$. For a scaling parameter $c \in 2^{\mathbb{N}}$, we define $u_{c}$ to be the solution of (1.1) with

$$
u_{c}(0)=c^{-d / 2} \varphi\left(\frac{x}{c}\right), \quad \dot{u}_{c}(0)=-i u_{c}(0) .
$$

Next we define as before $v_{c}=e^{i t} u_{c}$ and $w_{c}=P_{c}^{2 / \alpha} v_{c}$. Then $w_{c}$ solves (2.2) with

$$
w_{c}(0)=\varphi, \quad \dot{w}_{c}(0)=0 .
$$

Hence as $c \rightarrow \infty$

$$
E_{K}\left(u_{c}\right) \rightarrow\|\varphi\|_{L^{2}}^{2}
$$

By the assumption (1.15) and the invariance of $X$, we have

$$
\begin{aligned}
\limsup _{c \rightarrow \infty}\left\|\left(w_{c}\right)_{<c \delta}\right\|_{X} & =\limsup _{c \rightarrow \infty}\left\|\left(u_{c}\right)_{<\delta}\right\|_{X} \\
& \leq \limsup _{c \rightarrow \infty} C_{X}\left(E_{K}\left(u_{c}\right)\right)=C_{X}\left(M_{S}(\varphi)\right) .
\end{aligned}
$$

Moreover, by the convergence result in [18] for the non-relativistic limit, we have $w_{c} \rightarrow w$ in $C\left(\mathbb{R} ; H^{1}\right)$, where $w$ is the solution of NLS (1.2) with $w(0)=\varphi$. Hence we have $\left(w_{c}\right)_{<c \delta} \rightarrow w$ in $C\left(\mathbb{R} ; H^{1}\right)$, then by the Fatou property we have $w \in X$ and

$$
\|w\|_{X} \leq C_{X}\left(M_{S}(\varphi)\right) .
$$

Finally, we extend the above to the case $\varphi \in L^{2}$. For $n \in \mathbb{N}$, let $w_{n}$ be the solution of (1.2) with $w_{n}(0)=\varphi_{<n} \in H^{1}$. In the linear case $\mu=0$, it is clear that $w_{n}$ converges to the solution $w$ with initial data $w(0)=\varphi$ in $C\left(\mathbb{R} ; L^{2}\right)$, and then by the Fatou property we get the desired bound

$$
\|w\|_{X} \leq \liminf _{n \rightarrow \infty}\left\|w_{n}\right\|_{X} \leq \liminf _{n \rightarrow \infty} C_{X}\left(\left\|w_{n}\right\|_{L^{2}}^{2}\right)=C_{X}\left(M_{S}(w)\right)
$$

In the nonlinear case, we need to use the local wellposedness in the critical case. For that purpose we may set, by the assumption, $X=L_{t}^{p} L_{x}^{q}$ 
with $2<p<\infty$ and $2 / p+d / q=d / 2$, where we may avoid $p=2$ by interpolation with the trivial $L^{\infty} L^{2}$ bound.

Lemma 3.1 For any $\varphi \in L^{2}\left(\mathbb{R}^{d}\right)$, there exists a unique $T>0$ and a unique solution $w$ of $N L S(1.2)$ on $[0, T)$ such that $w \in C\left(\left[0, T^{\prime}\right] ; L^{2}\right) \cap$ $X\left(0, T^{\prime}\right)$ for any $T^{\prime} \in(0, T)$ and either $T=\infty$ or $\|w\|_{X(0, T)}=\infty$. If $\|w\|_{X(0, T)}<\infty$, then all the other Strichartz norms are also finite.

Moreover, for any sequence $\varphi_{n} \rightarrow \varphi$ converging in $L^{2}$, the corresponding solution $w_{n}$ exists on $\left[0, T^{\prime}\right]$ for large $n$ and $w_{n} \rightarrow w$ in $L^{\infty} L^{2} \cap X\left(0, T^{\prime}\right)$ for any $T^{\prime} \in(0, T)$.

Proof. We give the proof for the sake of completeness, though it is a well known fact. First we construct the solution on small interval $(0, S)$. Let $\|\varphi\|_{L^{2}}=Q, w^{0}:=e^{-i t \Delta / 2} \varphi$ and $\left\|w^{0}\right\|_{X(0, S)}=\varepsilon$. Define $Y:=L_{t}^{\rho} L_{x}^{\eta}$ by

$$
\frac{4}{d p}+\frac{1}{\rho}=1-\frac{1}{\rho}, \frac{4}{d q}+\frac{1}{\eta}=1-\frac{1}{\eta}
$$

Then we have $2<\rho<\infty$ and $2 / \rho+d / \eta=d / 2$. Define a sequence $w^{j}$ by

$$
w^{j+1}=w^{0}+\frac{1}{2 i} \int_{0}^{t} e^{-i(t-s) \Delta / 2} f\left(w^{j}(s)\right) d s .
$$

By the Strichartz estimate together with the Hölder inequality, we have

$$
\begin{array}{r}
\left\|w^{1}-w^{0}\right\|_{L^{\infty} L^{2} \cap X \cap Y(0, S)} \lesssim\left\|w^{0}\right\|_{Y(0, S)}\left\|w^{0}\right\|_{X(0, S)}^{4 / d} \lesssim Q \varepsilon^{4 / d}, \\
\left\|w^{j+1}-w^{j}\right\|_{L^{\infty} L^{2} \cap X \cap Y(0, S)} \\
\lesssim\left\|w^{j}-w^{j-1}\right\|_{Y(0, S)}\left\|\left(w^{j}, w^{j-1}\right)\right\|_{X(0, S)}^{4 / d} .
\end{array}
$$

Hence if $\varepsilon+Q \varepsilon^{4 / d} \ll 1$ is sufficiently small, then $w^{j} \rightarrow \exists w$ converges in $L^{\infty} L^{2} \cap X \cap Y(0, S)$, and $w$ solves NLS on $(0, S)$. The uniqueness follows from the same estimate as $w^{j+1}-w^{j}$ above. In addition, we get

$$
\begin{aligned}
& \|w\|_{L^{\infty} L^{2} \cap X \cap Y(0, S)} \lesssim Q, \\
& \left\|w-w^{0}\right\|_{L^{\infty} L^{2} \cap X \cap Y(0, S)} \lesssim\|w\|_{Y(0, S)}\|w\|_{X(0, S)}^{4 / d} .
\end{aligned}
$$

Now suppose that $\|w\|_{X(0, S)} \leq \varepsilon^{d / 4+1} \ll \varepsilon$. Then the above inequality implies

$$
\left\|w-w^{0}\right\|_{X(0, S)} \lesssim Q \varepsilon^{1+4 / d} \ll \varepsilon,
$$


thus $\left\|w^{0}\right\|_{X(0, S)} \ll \varepsilon$, contradicting the definition of $\varepsilon$. Hence

$$
\|w\|_{X(0, S)} \geq \varepsilon^{d / 4+1} .
$$

Since $\|w(t)\|_{L^{2}}=Q$ is preserved, we can repeat the above argument from $t=S$, and so on. Then either we get a global solution in finite steps, or infinite increasing sequence $0=S_{0}<S_{1}<\cdots$ such that $\|w\|_{X\left(S_{j}, S_{j+1}\right)} \geq$ $\varepsilon^{d / 4+1}$ with a fixed small $\varepsilon>0$. In the first case, we finish the proof with $T=\infty$, and all the Strichartz norms are finite. In the second case, we let $T=\lim _{j \rightarrow \infty} S_{j}$. Then $w$ is a unique solution on $(0, T)$ in $L^{\infty} L^{2} \cap X\left(0, T^{\prime}\right)$ for any $T^{\prime}<T$, and we have $\|w\|_{X(0, T)}=\infty$.

Finally we prove the continuity. Let $w_{n}^{0}=e^{-i t \Delta / 2} \varphi_{n}$. Then $w_{n}^{0} \rightarrow w^{0}$ in $L^{\infty} L^{2} \cap X \cap Y$, and hence for large $n$ the solution $w_{n}$ is obtained on $(0, S)$ by the above iteration. Moreover we have

$$
\begin{aligned}
& \left\|w_{n}-w\right\|_{L^{\infty} L^{2} \cap X \cap Y(0, S)} \\
& \lesssim\left\|w_{n}(0)-w(0)\right\|_{L^{2}}+\left\|w_{n}-w\right\|_{Y(0, S)}\left\|\left(w_{n}, w\right)\right\|_{X(0, S)}^{4 / d},
\end{aligned}
$$

and

$$
\left\|\left(w_{n}, w\right)\right\|_{X(0, S)}^{4 / d} \lesssim\left(\varepsilon+Q \varepsilon^{4 / d}\right)^{4 / d} \ll 1 .
$$

Hence

$$
\left\|w_{n}-w\right\|_{L^{\infty} L^{2} \cap X \cap Y(0, S)} \lesssim\left\|w_{n}(0)-w(0)\right\|_{L^{2}} \rightarrow 0 .
$$

We can repeat this argument to cover any interval $\left[0, T^{\prime}\right] \subset[0, T)$.

Now it is easy to finish the theorem. Let $w$ be the unique solution given by the above lemma and $T>0$ be the maximal existence time. Then for any $T^{\prime} \in(0, T)$, we have $w_{n} \rightarrow w$ in $X\left(0, T^{\prime}\right)$. Hence

$$
\|w\|_{X\left(0, T^{\prime}\right)}=\lim _{n \rightarrow \infty}\left\|w_{n}\right\|_{X\left(0, T^{\prime}\right)} \leq C_{X}\left(M_{S}(w)\right),
$$

and by Fatou's lemma for $T^{\prime} \rightarrow T-0$, we get $T=\infty$ and

$$
\|w\|_{X(0, \infty)} \leq C_{X}\left(M_{S}(w)\right) .
$$

Finally by the translation invariance in $t$, we obtain

$$
\|w\|_{X(\mathbb{R})} \leq C_{X}\left(M_{S}(w)\right),
$$

and moreover, $w_{n} \rightarrow w$ in $C\left(\mathbb{R} ; L^{2}\right)$. Since all the Strichartz norms are finite 
for $w$, it is quite standard to get the scattering result. As for the general $X$ in the theorem, we obtain the desired bound on $w$ by transfer from $w_{n}$ by the Fatou property.

\section{Proof for $H^{1}$ critical case}

We prove the results in the $H^{1}$ critical case, Theorems 1.5, 1.6 and 1.7. Since one of our goals (which is not yet achieved) is to give another proof for the global wellposedness of the NLS, we will not use that result in the arguments, except for the final step of Theorem 1.7, which is not related to our goals.

\subsection{General transfer}

Here we prove the transfer for general homogeneous spaces (Theorem 1.3). The idea is almost the same as in the $L^{2}$ critical case. Let $\varphi \in \dot{H}^{1}$, and for a scaling parameter $c \in 2^{\mathbb{N}}$, let $w_{c}$ be the solution of $(2.2)$ with the initial data

$$
w_{c}(0)=\varphi-\varphi_{<1 / c} \in H^{1}, \quad \dot{w}_{c}(0)=0 .
$$

Let $v_{c}=P_{1 / c}^{2 / \alpha} w_{c}$ and $u_{c}=e^{i t} v_{c}$. Then $u_{c}$ is the solution of NLKG (1.1) with

$$
\begin{aligned}
E_{K}^{\prime}\left(u_{c}\right) & =\frac{1}{2} \int_{\mathbb{R}^{d}}\left|\frac{\dot{w}_{c}}{c}\right|^{2}+\left|\nabla w_{c}\right|^{2}+F\left(w_{c}\right) d x \\
& =\frac{1}{2} \int\left|\nabla w_{c}(0)\right|^{2}+F\left(w_{c}(0)\right) d x<\infty, \\
M_{K}\left(u_{c}\right) & =c^{2} \int\left|w_{c}(0)\right|^{2} d x<\infty .
\end{aligned}
$$

Hence it is global and by the assumption

$$
\left\|\left(w_{c}\right)_{<c \delta}\right\|_{X}=\left\|\left(u_{c}\right)_{<\delta}\right\|_{X} \leq C_{X}\left(E_{K}^{\prime}\left(u_{c}\right)\right) .
$$

Next we consider the weak limit of $w_{c}$. By the bound on $E_{K}^{\prime}\left(u_{c}\right), w_{c}$ is uniformly bounded in $\dot{H}_{x}^{1}$. As for the time continuity, we use the equation for $w_{c}$ :

$$
\begin{aligned}
\underset{S}{T} & =\int_{S}^{T} \dot{w}_{c}(t) d t=\frac{i}{2} \int_{S}^{T} c^{-2} \ddot{w}_{c}-\Delta w_{c}-f\left(w_{c}\right) d t \\
& =\frac{i}{2}\left\{\left[c^{-2} \dot{w}_{c}\right]_{S}^{T}-\int_{S}^{T} \Delta w_{c}+f\left(w_{c}\right) d t\right\} .
\end{aligned}
$$


Putting the first and the third expressions into the norms, we get

$$
\begin{aligned}
\left\|\left[w_{c}\right]_{S}^{T}\right\|_{L^{2}} & \leq \int_{S}^{T}\left\|\dot{w}_{c}(t)\right\|_{L^{2}} d t \lesssim|T-S| c E_{K}^{\prime}\left(u_{c}\right)^{1 / 2} \\
\left\|\left[w_{c}\right]_{S}^{T}\right\|_{H^{-1}} & \lesssim\left\|c^{-2}\left[\dot{w}_{c}\right]_{S}^{T}\right\|_{L^{2}}+\int_{S}^{T}\left\|\Delta w_{c}\right\|_{\dot{H}^{-1}}+\left\|f\left(w_{c}\right)\right\|_{L^{2^{*}}} d t \\
& \lesssim c^{-1}+|T-S|\left(E_{K}^{\prime}\left(u_{c}\right)^{1 / 2}+E_{K}^{\prime}\left(u_{c}\right)^{\alpha+1}\right) .
\end{aligned}
$$

Using the upper estimate for $c \sqrt{|T-S|}<1$ and otherwise the lower one, we deduce that $w_{c}$ is uniformly bounded in $C^{1 / 2}\left(\mathbb{R} ; H^{-1}\right)$. Then using the standard compactness argument, we get a subsequence of $c \rightarrow \infty$ where $w_{c}$ converges in $C\left(\mathbb{R} ; w-\dot{H}^{1} \cap L_{\text {loc }}^{p}\right)$, for any $p<2^{*}$, and hence the limit $w \in$ $C\left(\mathbb{R} ; w-\dot{H}^{1}\right)$ is a weak solution of $(1.2)$. Then we have also $\left(w_{c}\right)_{<c \delta} \rightarrow w$ in $C\left(\mathbb{R} ; w-\dot{H}^{1}\right)$, and by the Fatou property of $X$, we have $w \in X$ with

$$
\begin{aligned}
\|w\|_{X} & \leq \limsup _{c \rightarrow \infty}\left\|\left(w_{c}\right)_{<c \delta}\right\|_{X} \\
& \leq \limsup _{c \rightarrow \infty} C_{X}\left(E_{K}^{\prime}\left(u_{c}\right)\right)=C_{X}\left(E_{S}(w ; 0)\right) .
\end{aligned}
$$

Next, if we can choose $X=L_{t}^{p} \dot{H}_{q, x}^{1}$ with $2 \leq p<\infty$, then it coincides with the unique local solution $w^{\prime} \in C\left([0, T] ; \dot{H}^{1}\right)$ with the full Strichartz norms on $[0, T]$, which is constructed by the iteration. The reason is as follows. First we can assume that $1 / p<1 / 2-1 /(2 d)$ by interpolating with the $L_{t}^{\infty} \dot{H}^{1}$ bound. Then we have $q<d$. For $0<S<T$, we estimate the difference by the endpoint Strichartz

$$
\left\|w-w^{\prime}\right\|_{L^{2} L^{2^{*}(0, S)}} \lesssim\left\|\left(w, w^{\prime}\right)\right\|_{L^{p} \dot{H}_{q}^{1}(0, S)}^{4 /(d-2)}\left\|w-w^{\prime}\right\|_{L^{2} L^{2^{*}(0, S)}},
$$

where we used the embedding $\dot{H}_{q}^{1} \subset L^{q d /(q-d)}$. Since $p<\infty$, the $L^{p} \dot{H}_{q}^{1}$ norm tends to 0 for both $w$ and $w^{\prime}$, and the $L^{2} L^{2^{*}}$ norm is finite by the Sobolev embedding, $w=w^{\prime}$ on such $(0, S)$. Repeating the same argument from $S$, we get $w=w^{\prime}$ as long as they are in $L^{p} \dot{H}_{q}^{1}$, so $w^{\prime}$ is also global. For any $\varepsilon>0$, there is $T>0$, such that $\|w\|_{L^{p} \dot{H}_{q}^{1}(T, \infty)}<\varepsilon$, because $p<\infty$. Then by the same estimate as above, we get

$$
\left\|w-w^{T}\right\|_{L^{2} \dot{H}_{2^{*}}^{1}(T, \widetilde{T})} \lesssim \varepsilon^{4 /(d-2)}\|w\|_{L^{2} \dot{H}_{2^{*}}^{1}(T, \widetilde{T})},
$$

where $w^{T}=e^{-i(t-T) \Delta / 2} w(T)$. Hence if $\varepsilon>0$ is small enough, we have

$$
\|w\|_{L^{2} \dot{H}_{2^{*}}^{1}(T, \widetilde{T})} \leq 2\left\|w^{T}\right\|_{L^{2} \dot{H}_{2^{*}}^{1}(T, \widetilde{T})} \lesssim\|w(T)\|_{\dot{H}^{1}} .
$$


By the limit $\widetilde{T} \rightarrow \infty$, we deduce that $w \in L^{2} \dot{H}_{2^{*}}^{1}(0, \infty)$. Now that $w$ has by interpolation all the Strichartz norms (of $\dot{H}^{1}$ regularity) finite, it is quite standard to get the scattering in $\dot{H}^{1}$.

\subsection{Transfer of dyadic transition}

Now we turn to the specific norm of the dyadic transition. First we prove Theorem 1.5 by the non-relativistic limit. In this case, the norm is not scaling invariant, but it converges to the Schrödinger counterpart.

Proof of Theorem 1.5. Let $w_{c}, v_{c}$ and $u_{c}$ with $c \in 2^{\mathbb{N}}$ as in the previous section. Let $u_{c}^{T}$ and $w_{c}^{T}$ be the free solutions sharing the same data at $t=T$ with $u_{c}$ and $w_{c}$ respectively. Then we have

$$
\begin{aligned}
C\left(E_{K}^{\prime}\left(u_{c}\right)\right)^{p} & \geq\left\|\left(u_{c}\right)_{<\delta}\right\|_{S_{1}^{p} L^{2^{*}}}^{p} \\
& =\sup _{ \pm} \sup _{T \in \mathbb{R}} \sum_{j \in \mathbb{Z}} \sup _{2^{j}<t<2^{j+1}}\left\|\left[w_{c}-w_{c}^{T}\right]_{<c \delta}(T \pm t)\right\|_{L_{x}^{2^{*}}}^{p}
\end{aligned}
$$

By the same argument as in the previous section, we get a subsequence $c \rightarrow$ $\infty$ along which $w_{c} \rightarrow w$ in $C\left(\mathbb{R} ; w-\dot{H}^{1} \cap L_{\text {loc }}^{p}\right)$ for $p<2^{*}$, where $w$ is a weak solution of NLS. Then $w_{c}^{T}$ also converges to the free Schrödinger solution $w^{T}$ in $C\left(\mathbb{R} ; w-\dot{H}^{1}\right)$ for each $T \in \mathbb{R}$. By the embedding $\dot{H}^{1} \subset L^{2^{*}}$ together with the Fatou property of $L^{p}$ for $p>1$, the above bound transfers into

$$
\|w\|_{S_{\infty}^{p} L^{2^{*}}} \leq \limsup _{c \rightarrow \infty} C\left(E_{K}^{\prime}\left(u_{c}\right)\right)=C\left(E_{S}(w ; 0)\right) .
$$

It remains to prove that $w$ is the strong solution. First, the above $S_{\infty}^{p} L^{2^{*}}$ bound implies that for any $T \in \mathbb{R}$

$$
\lim _{t \rightarrow 0}\left\|w(T+t)-w_{T}(T+t)\right\|_{L^{2^{*}}}=0,
$$

because $p<\infty$. Hence $w \in C\left(\mathbb{R} ; L^{2^{*}}\right)$. We show that such a solution coincides with the local strong solution constructed by the Strichartz estimate, starting from any time. The idea for showing the uniqueness is essentially in [23], though our situation is easier. By the time translation invariance, it suffices to start with $t=0$. Let $w^{\prime}$ be the local strong solution given by the standard iteration with an existence time $T>0$, and let $w=w^{\prime}+\gamma$. Then we have

$$
\gamma(t)=\frac{1}{2 i} \int_{0}^{t} e^{-i(t-s) \Delta / 2}\left[f\left(w^{\prime}+\gamma\right)-f\left(w^{\prime}\right)\right](s) d s,
$$


for $0<t<T$. Since $\left|f\left(w^{\prime}+\gamma\right)-f\left(w^{\prime}\right)\right| \lesssim|\gamma|\left(\left|w^{\prime}\right|+|\gamma|\right)^{2^{*}-2}$, we have by the Strichartz estimate,

$$
\begin{aligned}
\|\gamma\|_{L_{t}^{2} L_{x}^{2 *}} & \lesssim\left\|\left(w^{\prime}\right)^{2^{*}-2} \gamma\right\|_{L_{t}^{p_{1}} L_{x}^{q_{1}}}+\left\|\gamma^{2^{*}-1}\right\|_{L_{t}^{2} L_{x}^{2 *}} \\
& \lesssim\left[\left\|w^{\prime}\right\|_{L_{t, x}^{p_{3}}}+\|\gamma\|_{L_{t}^{\infty} L_{x}^{2^{*}}}\right]^{2^{*}-2}\|\gamma\|_{L_{t}^{2} L_{x}^{2^{*}}},
\end{aligned}
$$

on any time interval $(0, S) \subset(0, T)$, where $2_{*}=2 d /(d+2), p_{3}=2(d+$ $2) /(d-2)$,

$$
\begin{aligned}
\left(\frac{1}{p_{1}}, \frac{1}{q_{1}}\right) & =\left(\frac{1}{2}, \frac{1}{2^{*}}\right)+\frac{2^{*}-2}{p_{3}}(1,1) \\
& =\left(\frac{d+6}{2(d+2)}, \frac{d^{2}+4 d-4}{2 d(d+2)}\right) .
\end{aligned}
$$

Since $\lim _{t \rightarrow 0}\|\gamma(t)\|_{L^{2^{*}}}=0$ and $w^{\prime} \in L_{t, x}^{p_{3}}$, the right hand side of (4.14) becomes much smaller than the left as $S \rightarrow+0$, unless $\|\gamma\|_{L^{2} L^{2^{*}(0, S)}}=0$. Hence $w=w^{\prime}$ near $t=0$. Since the above argument works around any time, $w$ is the unique strong solution, and moreover global. Namely, we have the global wellposedness for the NLS. In particular, $w$ has the Strichartz bounds locally in time, and the energy $E_{S}(w)$ is conserved in time.

Moreover, we get the scattering result as follows. Since the free solution satisfies $\left\|w_{0}(t)\right\|_{L^{2^{*}}} \rightarrow 0$ as $t \rightarrow \infty$ (it is easily seen by approximating the initial data by $C_{0}^{\infty}$ functions), we also have $\|w(t)\|_{L^{2^{*}}} \rightarrow 0$ by the $S_{\infty}^{p} L^{2^{*}}$ bound. Then for $T>S \gg 1$ we have by the Strichartz,

$$
\begin{aligned}
\left\|\left[e^{i t \Delta / 2} w(t)\right]_{S}^{T}\right\|_{\dot{H}^{1}} & +\left\|w-w_{S}\right\|_{L^{2} \dot{H}_{2^{*}}^{1}(S, T)} \\
& \lesssim\|f(w)\|_{L_{t}^{2} \dot{H}_{2_{*}^{*}}^{1}(S, T)} \\
& \lesssim\|w\|_{L^{2} \dot{H}_{2^{*}}^{1}(S, T)}\|w\|_{L_{t}^{\infty} L_{x}^{2^{*}}(S, T)}^{2^{*}-2}
\end{aligned}
$$

Since the last norm tends to 0 as $S \rightarrow \infty$, and $w_{S}$ is bounded in $L^{2} \dot{H}_{2^{*}}^{1}$, we deduce that $w$ is bounded in $L^{2} \dot{H}_{2^{*}}^{1}(S, T)$ for sufficiently large $S$. This implies also that the other Strichartz norms of $\dot{H}^{1}$ level are globally bounded. Then the above inequality implies that the left hand side tends to 0 as $S \rightarrow$ $\infty$, which gives the scattering for $w$.

In the rest of the section, we prove linear estimates for the $S_{*}^{p} L^{2^{*}}$ norms, from which Theorems 1.6 and 1.7 will follow. 
Lemma 4.1 Let $d \geq 3$. Assume $u(t, x), w(t, x)$ and $F(t, x)$ satisfy

$$
\ddot{u}-\Delta u+u=2 i \dot{w}-\Delta w=F
$$

on $\mathbb{R}^{1+d}$. Then we have

$$
\begin{aligned}
& \left\|u_{<1}\right\|_{S_{1}^{2} B_{2^{*}, 2}^{0}}+\left\|\dot{u}_{<1}\right\|_{S_{1}^{2} B_{2^{*}, 2}^{0}} \lesssim\left\|F_{<1}\right\|_{L_{t}^{2} \dot{B}_{2_{*}, 2}^{1}}, \\
& \|w\|_{S_{1}^{2} B_{2^{*}, 2}^{0}} \lesssim\|F\|_{L_{t}^{2} \dot{B}_{2 *, 2}^{1}},
\end{aligned}
$$

where $2_{*}:=2 d /(d+2)$ denotes the dual Sobolev exponent.

The norm on the right is the dual endpoint Strichartz on the $\dot{H}^{1}$ level for the Schrödinger equation, which is valid on the restricted frequency $|\xi| \lesssim$ 1 for the Klein-Gordon as well. We have the embedding $B_{2^{*}, 2}^{0} \subset L^{2^{*}}$ and $\dot{B}_{2_{*}, 2}^{1} \supset \dot{H}_{2_{*}}^{1}$.

Proof. First of all, the above estimate for the Klein-Gordon is transferred to the Schrödinger by the same argument as in the above proof of Theorem 1.5, because the norm of $F$ has the right scaling property. Hence it suffices to prove the Klein-Gordon case. However, it is indeed more complicated because of the mixed scaling present in the equation.

By the symmetry for the time inversion and translation, it suffices to bound the sup for $T=0$ and $t>0$. Since the semi-norms are trivial on the free solutions, we may assume that $u(0)=\dot{u}(0)=0$. Moreover, we can decompose $u$ and $F$ dyadically in the frequency

$$
u=\sum_{k \in \mathbb{Z}} u_{k}, \quad F=\sum_{k \in \mathbb{Z}} F_{k},
$$

where $u_{k}:=u_{<2^{k}}-u_{<2^{k-1}}$. Then by the Minkowski inequality, it suffices to give uniform estimate for each $k \leq 0$.

Let $v:=e^{i t} u$ and $F^{\prime}:=e^{i t} F$. Then after the dyadic decomposition we have

$$
\ddot{v}_{k}+2 i \dot{v}_{k}-\Delta v_{k}=F_{k}^{\prime} .
$$

Note that

$$
\left|\left(u_{k}, \dot{u}_{k}\right)\right| \sim\left|\left(v_{k}, \dot{v}_{k}\right)\right|,
$$

as vectors at every $t, x, k$.

Now we start estimation with smaller $j$. Let $\widetilde{I}_{j}:=\left(0,2^{j+1}\right)$. By inte- 
gration in $t$, we have

$$
\left\|\left(v_{k}, \dot{v}_{k}\right)\right\|_{L_{t}^{\infty} L_{x}^{2^{*}}\left(\widetilde{I}_{j}\right)} \leq\left\|\left(\dot{v}_{k}, \ddot{v}_{k}\right)\right\|_{L_{t}^{1} L_{x}^{2 *}\left(\widetilde{I}_{j}\right)},
$$

and the right hand side is bounded by the Sobolev and the equation

$$
\begin{aligned}
\left\|\dot{v}_{k}\right\|_{L_{t}^{1} L_{x}^{2 *}\left(\widetilde{I}_{j}\right)} & \lesssim 2^{j+k}\left\|\dot{v}_{k}\right\|_{L_{t}^{\infty} L^{2}}, \\
\left\|\ddot{v}_{k}\right\|_{L_{t}^{1} L_{x}^{2 *}\left(\widetilde{I}_{j}\right)} & \lesssim 2^{j+k}\left\|\dot{v}_{k}\right\|_{L_{t}^{\infty} L^{2}}+2^{j+2 k}\left\|v_{k}\right\|_{L_{t}^{\infty} \dot{H}^{1}} \\
& +2^{j / 2+2 k}\left\|F_{k}\right\|_{L_{t}^{2} \dot{H}^{-1}} .
\end{aligned}
$$

Thus we get

$$
\begin{aligned}
\sum_{j \leq-k}\left\|\left(v_{k}, \dot{v}_{k}\right)\right\|_{L_{t}^{\infty} L_{x}^{2^{*}}\left(I_{j}\right)} & \\
& \lesssim\left\|\dot{v}_{k}\right\|_{L_{t}^{\infty} L_{x}^{2}}+2^{k}\left\|v_{k}\right\|_{L_{t}^{\infty} \dot{H}_{x}^{1}}+2^{3 k / 2}\left\|F_{k}\right\|_{L_{t}^{2} \dot{H}^{-1}} .
\end{aligned}
$$

Next we consider the sum over $-k<j \leq-2 k$. By using the equation

$$
2 i v_{k}=-\dot{v}_{k}+\int_{0}^{t}\left(\Delta v_{k}+F_{k}^{\prime}\right)(s) d s
$$

we have

$$
\begin{aligned}
& \left\|\left(v_{k}, \dot{v}_{k}\right)\right\|_{L_{t}^{\infty} L_{x}^{2^{*}\left(\widetilde{I}_{j}\right)}} \lesssim\left\|\dot{v}_{k}\right\|_{L_{t}^{\infty} L_{x}^{2^{*}}}+\left\|\Delta v_{k}+F_{k}^{\prime}\right\|_{L_{t}^{1} L_{x}^{2^{*}}\left(\widetilde{I}_{j}\right)} \\
& \lesssim 2^{k}\left\|\dot{v}_{k}\right\|_{L^{\infty} L^{2}}+2^{j+2 k}\left\|v_{k}\right\|_{L_{t}^{\infty} L_{x}^{2^{*}}}+2^{j / 2+2 k}\left\|F_{k}\right\|_{L_{t}^{2} \dot{H}_{x}^{-1}}
\end{aligned}
$$

Summing it over $-k<j \leq-2 k$, and adding (4.24), we get

$$
\begin{aligned}
\sum_{j \leq-2 k}\left\|\left(v_{k}, \dot{v}_{k}\right)\right\|_{L_{t}^{\infty} L_{x}^{2^{*}}\left(I_{j}\right)} & \\
& \lesssim\left\|\dot{v}_{k}\right\|_{L_{t}^{\infty} L_{x}^{2}}+\left\|v_{k}\right\|_{L_{t}^{\infty} L_{x}^{2^{*}}}+2^{k}\left\|F_{k}\right\|_{L_{t}^{2} \dot{H}^{-1}} .
\end{aligned}
$$

Finally we consider the sum over $-2 k<j$. We use the identity

$$
\partial_{t}\left[\left|u_{k}\right|^{2}+\left|\dot{u}_{k}\right|^{2}\right]=2\left\langle\dot{u}_{k}, \ddot{u}_{k}+u_{k}\right\rangle=2\left\langle\dot{v}_{k}+i v_{k}, \Delta v_{k}+F_{k}^{\prime}\right\rangle,
$$

Integrating in $t$, we get

$$
\operatorname{osc}_{I_{j}}\left(\left|u_{k}\right|^{2}+\left|\dot{u}_{k}\right|^{2}\right) \leq 2\left\|\dot{v}_{k}+i v_{k}\right\|_{L_{t}^{2}\left(I_{j}\right)}\left\|\Delta v_{k}+F_{k}^{\prime}\right\|_{L_{t}^{2}\left(I_{j}\right)},
$$

where we denote

$$
\operatorname{osc}_{I} f(t)=\sup _{t \in I} f(t)-\inf _{t \in I} f(t)
$$


Integrating in $x$, we get

$$
\begin{aligned}
\operatorname{osc}_{I_{j}} \|\left(u_{k}, \dot{u}_{k}\right) & \|_{L_{x}^{2 *}}^{2} \\
& \lesssim\left\|\dot{v}_{k}+i v_{k}\right\|_{L_{x}^{2} L_{t}^{2}\left(I_{j}\right)}\left\|\Delta v_{k}+F_{k}^{\prime}\right\|_{L_{x}^{2} L_{t}^{2}\left(I_{j}\right)} .
\end{aligned}
$$

Hence we obtain

$$
\sum_{j \in \mathbb{Z}} \operatorname{osc}_{I_{j}}\left\|\left(u_{k}, \dot{u}_{k}\right)\right\|_{L_{x}^{2^{*}}}^{2} \lesssim\left\|\dot{v}_{k}+i v_{k}\right\|_{L_{t}^{2} L_{x}^{2 *}}\left\|\Delta v_{k}+F_{k}^{\prime}\right\|_{L_{t}^{2} L_{x}^{2 *}}
$$

The right hand side can be estimated by the Strichartz

$$
\left\|\dot{v}_{k}\right\|_{L_{t}^{2} L_{x}^{2^{*}}}+2^{k}\left\|v_{k}\right\|_{L_{t}^{2} L_{x}^{2 *}} \lesssim 2^{k}\left\|F_{k}\right\|_{L_{t}^{2} L_{x}^{2 *}}
$$

and by the Sobolev

$$
\left\|F_{k}\right\|_{L_{t}^{2} L_{x}^{2^{*}}} \lesssim 2^{2 k}\left\|F_{k}\right\|_{L_{t}^{2} L_{x}^{2 *}}
$$

Thus we obtain for $k \leq 0$,

$$
\sum_{j \in \mathbb{Z}} \operatorname{osc}_{I_{j}}\left\|\left(u_{k}, \dot{u}_{k}\right)\right\|_{L_{x}^{2^{*}}} \lesssim 2^{2 k}\left\|F_{k}\right\|_{L_{t}^{2} L_{x}^{2 *}}
$$

On the other hand we have

$$
\left|I_{j}\right|^{1 / 2} \inf _{I_{j}}\left\|\left(u_{k}, \dot{u}_{k}\right)\right\|_{L_{x}^{2^{*}}} \leq\left\|\left(v_{k}, \dot{v}_{k}\right)\right\|_{L_{t}^{2} L_{x}^{2^{*}}},
$$

Applying the Strichartz on the right, we get

$$
\inf _{I_{j}}\left\|\left(u_{k}, \dot{u}_{k}\right)\right\|_{L_{x}^{2^{*}}} \lesssim 2^{-j / 2}\left\|F_{k}\right\|_{L_{t}^{2} L_{x}^{2 *}}
$$

Summing over $j+2 k \geq 0$,

$$
\sum_{j \geq 2 k} \inf _{I_{j}}\left\|\left(u_{k}, \dot{u}_{k}\right)\right\|_{L_{x}^{2^{*}}} \lesssim 2^{k}\left\|F_{k}\right\|_{L_{t}^{2} L_{x}^{2 *}}
$$

Adding it to (4.35),

$$
\sum_{j \geq 2 k} \sup _{I_{j}}\left\|\left(u_{k}, \dot{u}_{k}\right)\right\|_{L_{x}^{2^{*}}}^{2} \lesssim 2^{2 k}\left\|F_{k}\right\|_{L_{t}^{2} L_{x}^{2 *}}^{2} .
$$

Summing it with (4.27), we obtain

$$
\begin{aligned}
\left\|\left(u_{k}, \dot{u}_{k}\right)\right\|_{\ell_{j}^{2} L_{t}^{\infty} L_{x}^{2^{*}}\left(I_{j}\right)} & \\
& \lesssim\left\|v_{k}\right\|_{L_{t}^{\infty} L_{x}^{2 *}}+\left\|\dot{v}_{k}\right\|_{L_{t}^{\infty} L_{x}^{2}}+2^{k}\left\|F_{k}\right\|_{L_{t}^{2} L_{x}^{2 *}}
\end{aligned}
$$


Since the first two terms on the right are bounded by the last one through the Strichartz estimate, we get the desired estimate.

Proof of Theorem 1.6. By the results in [20, 21], we can bound the nonlinearity in some appropriate dual Strichartz norms in terms of the energy $E_{K}(u)$. Then by the Strichartz estimate we get (in the following $C\left(E_{K}(u)\right)$ denotes arbitrary continuous functions of $\left.E_{K}(u)\right)$

$$
\|u\|_{L_{t}^{2} L_{x}^{2 *}} \leq C\left(E_{K}(u)\right)
$$

then by the Hölder and Sobolev inequalities,

$$
\|f(u)\|_{L_{t}^{2} L_{x}^{2 *}} \lesssim\|u\|_{L_{t}^{\infty} L_{x}^{\alpha d / 2}}^{\alpha}\|u\|_{L_{t}^{2} L_{x}^{2^{*}}} \leq C\left(E_{K}(u)\right),
$$

where we can use $H_{x}^{1} \subset L_{x}^{\alpha d / 2}$, because $4 / d \leq \alpha \leq 4 /(d-2)$ implies $2 \leq \alpha d / 2 \leq 2^{*}$. It is much stronger than what is required in the above lemma.

Theorem 1.7 is proved in the same way using the above lemma and uniform bound on the Strichartz norm, which was first proved in [3] for $d=3$, which is essentially valid as far as $d \geq 3$ and $4 / d<\alpha<4 /(d-2)$. The case $\alpha=4 /(d-2)$ was solved in $[6,24,31]$.

\section{References}

[1] Bahouri H. and Shatah J., Global estimate for the critical semilinear wave equation. Ann. Inst. H. Poincare Anal. Non Lineaire (6) 15 (1998), 783-789.

[2] Bergh J. and Löfström J., Interpolation spaces. An introduction. Grundlehren Math. Wiss. vol. 223, Springer, Berlin-Heiderberg-New York, 1976.

[3] Bourgain J., Scattering in the energy space and below for 3D NLS. J. Anal. Math. 75 (1998), 267-297.

[4] Bourgain J., Global wellposedness of defocusing critical nonlinear Schrödinger equation in the radial case. J. Amer. Math. Soc. (1) 12 (1999), 145-171.

[5] Colliander J., Grillakis M. and Tzirakis N., Improved interaction Morawetz inequalities for the cubic nonlinear Schrödinger equation on $\mathbb{R}^{2}$. arXiv: math/0703606.

[6 ] Colliander J., Keel M., Staffilani G., Takaoka H. and Tao T., Global well-posedness and scattering for energy-critical nonlinear Schödinger equations in $\mathbb{R}^{3}$. to appear in Ann. of Math.

[ 7 ] Colliander J., Keel M., Staffilani G., Takaoka H. and Tao T., A refined global wellposedness result for Schrödinger equations with derivative. SIAM J. Math. Anal. (1) 34 (2002), 64-86. 
[ 8 ] Colliander J., Keel M., Staffilani G., Takaoka H. and Tao T., Almost conservation laws and global rough solutions to a nonlinear Schrödinger equation. Math. Res. Lett. (5-6) 9 (2002), 659-682.

[9] De Silva D., Pavlović N., Staffilani G. and Tzirakis N., Global well-posedness and polynomial bounds for the defocusing $L^{2}$-critical nonlinear Schrödinger equation in $\mathbb{R}$. to appear in Comm. Partial Differential Equations.

[10] De Silva D., Pavlović N., Staffilani G. and Tzirakis N., Global Well-Posedness for the $L^{2}$-critical nonlinear Schrödinger equation in higher dimensions. to appear in Comm. Pure Appl. Math.

[11] Fang Y. F. and Grillakis M. G., On the global existence of rough solutions of the cubic defocusing Schrödinger equation in $\mathbb{R}^{2+1}$. J. Hyperbolic Differ. Equ. (2) 4 (2007), 233-257.

[12] Ginibre J. and Velo G., Time decay of finite energy solutions of the non linear Klein-Gordon and Schrödinger equations. Ann. Inst. Henri. Poincaré 43 (1985), 399-442.

[13] Ginibre J., Soffer A. and Velo G., The global Cauchy problem for the critical nonlinear wave equation. J. Funct. Anal. 110 (1992), 96-130.

[14] Grillakis M., On nonlinear Schrödinger equations. Comm. Partial Differential Equations (9-10) 25 (2000), 1827-1844.

[15] Killip R., Tao T. and Visan M., The cubic nonlinear Schrödinger equation in two dimensions with radial data. math: AP/0707.3188.

[16] Killip R., Visan M. and Zhang X., The mass-critical nonlinear Schrödinger equation with radial data in dimensions three and higher. math: AP/0708.0849.

[17] Machihara S., Nakamura M., Nakanishi K. and Ozawa T., Endpoint Strichartz estimates and global solutions for the nonlinear Dirac equation. J. Funct. Anal. (1) 219 (2005), 1-20

[18] Machihara S., Nakanishi K. and Ozawa T., Nonrelativistic limit in the energy space for nonlinear Klein-Gordon equations. Math. Ann. (3) 322 (2002), 603-621.

[19] Masmoudi N., and Planchon F., On uniqueness for the critical wave equation. Comm. Partial Differential Equations (7-9) 31 (2006), 1099-1107

[20] Nakanishi K., Scattering theory for the nonlinear Klein-Gordon equation with Sobolev critical power. Internat. Math. Res. Notices 1 (1999), 31-60.

[21] Nakanishi K., Energy scattering for nonlinear Klein-Gordon and Schrödinger equations in spatial dimensions 1 and 2. J. Funct. Anal. (1) 169 (1999), 201-225.

[22] Nakanishi K., Remarks on the energy scattering for nonlinear Klein-Gordon and Schrödinger equations. Tohoku Math. J. (2) 53 (2001), 285-303.

[23] Planchon F., On uniqueness for semilinear wave equations. Math. Z. (3) 244 (2003), 587-599.

[24] Ryckman E. and Visan M., Global well-posedness and scattering for the defocusing energy-critical nonlinear Schrödinger equation in $\mathbb{R}^{1+4}$. Amer. J. Math. (1) 129 (2007), 1-60.

[25] Shatah J. and Struwe M., Well-posedness in the energy space for semilinear wave equations with critical growth. IMRN 7 (1994), 303-309. 
[26] Struwe M., Uniqueness for critical nonlinear wave equations and wave maps via the energy inequality. Comm. Pure Appl. Math. (9) 52 (1999), 1179-1188.

[27] Tao T., Spherically averaged endpoint Strichartz estimates for the two-dimensional Schrödinger equation. Comm. Partial Differential Equations (7-8) 25 (2000), 14711485 .

[28] Tao T., Global well-posedness and scattering for the higher-dimensional energycritical nonlinear Schrödinger equation for radial data. New York J. Math. 11 (2005), 57-80.

[29] Tsutsumi Y., $L^{2}$ solutions to nonlinear Schrödinger equations and nonlinear groups. Funk. Ekva. 30 (1987), 115-125.

[30] Tzirakis N., The Cauchy problem for the semilinear quintic Schrödinger equation in one dimension. Differential and Integral Equations 18 (2005), 947-960.

[31] Visan M., The defocusing energy-critical nonlinear Schrödinger equation in higher dimensions. Duke Math. J. (2) 138 (2007), 281-374.

Department of Mathematics

Kyoto University

Kyoto 606-8502, Japan

E-mail: n-kenji@math.kyoto-u.ac.jp 